\title{
Photoreflectance of Epitaxial InGaAs Quantum Rods
}

\author{
R. Nedzinskas ${ }^{a, *}$, V. Karpus ${ }^{a}$, B. ČechaviČius ${ }^{a}$, J. Kavaliauskas $^{a}$, G. VAlušis $^{a}$, \\ L.H. Li ${ }^{b}$, S.P. KHANNA ${ }^{b}$ AND E.H. LINFIELD ${ }^{b}$ \\ ${ }^{a}$ Semiconductor Physics Institute, Center for Physical Sciences and Technology \\ A. Goštauto 11, LT-01108, Vilnius, Lithuania \\ ${ }^{b}$ School of Electronic and Electrical Engineering, University of Leeds, Leeds LS2 9JT, United Kingdom

\begin{abstract}
Photoreflectance spectroscopy and photoluminescence have been used to study the optical properties and electronic structure of InGaAs quantum rods grown by molecular beam epitaxy. Spectral features associated with interband optical transitions localized in the quantum rod and the surrounding quantum well regions are examined. Experimental results are compared with calculations performed within the envelope function approximation. A red shift of the quantum rod- and a blue shift of the quantum well-related optical transitions, along with a significant increase in PL intensity have been observed if an As s source is used instead of an As source during the molecular beam epitaxial growth.
\end{abstract}

PACS: 78.67.Hc, 78.55.Cr, 81.05.Ea

\section{Introduction}

Recent interest on self-assembling quantum dots (QDs) as functional elements for semiconductor optical amplifiers (SOAs) has stimulated technological developments to improve QD homogeneity and polarization insensitivity. The typically flat-shape of the Stranski-Krastanow (SK) QDs permits the use of only transverse-electric (TE) polarized light, restricting their applicability in SOAs. To modify the polarization-dependent optical gain function, QD shape and composition engineering has been proposed [1]. Columnar QDs, or quantum rods (QRs), were realized by depositing a short period InAs/GaAs superlattice (SL) on top of a seed SK-grown QD layer. By careful choice of the growth parameters, QRs with aspect ratio (height/diameter) well exceeding 1 were then obtained [1]. Very recently it was demonstrated both theoretically [2] and experimentally [3] that an indium composition contrast between the $\operatorname{In}_{x} \mathrm{Ga}_{1-x} \mathrm{As}$ QR and surrounding $\operatorname{In}_{x} \mathrm{Ga}_{1-x}$ As layer, or quantum well $(\mathrm{QW}), x_{\mathrm{QR}} / x_{\mathrm{QW}}>3$ is needed to alter the polarization properties in favour of the TM mode. Such elongated nanostructures, following optimization of both their composition and geometry, already appear very attractive for fabrication of polarization-insensitive SOAs.

In this work, we have used photoreflectance (PR) spectroscopy to obtain further information on the optoelectronic properties of QR structures, and allow their growth to be optimized. This technique has already been proven to be well-suited to the characterization

* corresponding author; e-mail: ramunas@pfi.lt of doped quantum wells [4], and QD-superlattice systems [5]. Spectral features associated with interband optical transitions localized in the QR and surrounding two-dimensional InGaAs QW are discussed, and the experimental results are compared with the calculations performed within the envelope function approximation. We also study the influence of the arsenic source $\left(\mathrm{As}_{2}\right.$ or $\mathrm{As}_{4}$ ) on the observed spectral features.

\section{Samples and experimental details}

In our work, two nearly identical structures — samples L396 and L402 grown by molecular beam epitaxy (MBE) - are studied. They differ only in the arsenic source used: L396 was grown with $\mathrm{As}_{4}$ molecules, whereas L402 was grown with $\mathrm{As}_{2}$. In both cases (001)-oriented semi-insulating GaAs substrates [1] were used. After a GaAs buffer layer, $200 \mathrm{~nm}$ thick $\mathrm{Al}_{0.2} \mathrm{Ga}_{0.8} \mathrm{As}$ and $100 \mathrm{~nm}$ thick GaAs layers were grown. Self-assembled QRs were then formed by first depositing a 1.8 monolayer (ML) InAs QD seed layer followed by a 20 period GaAs/InAs (3 ML/0.64 ML) SL. The whole structure was completed by a $100 \mathrm{~nm}$ thick GaAs cap layer. Selective adatom incorporation into the strained areas on top of the seed QDs results in tall, indium-rich columns (i.e. QRs) within the indium-reduced InGaAs matrix. The QR height is controlled explicitly by the GaAs and InAs layer thicknesses, and the number of periods in the SL. In our case, an $N=20$ period SL leads to formation of QRs, which are $32 \mathrm{~nm}$ in height, and have an aspect ratio of 3.2 and an areal density of $1.7 \times 10^{10} \mathrm{~cm}^{-2}$ [1]. From transmission electron microscopy images, indium contents of $x_{\mathrm{QW}} \approx 0.16$ and $x_{\mathrm{QR}} \approx 0.45$ were estimated [6]. 
QR structures were measured using both PR and PL, allowing the study of the optical interband transitions within the structures at room temperature. A $\mathrm{He}-\mathrm{Ne}$ $(632.8 \mathrm{~nm})$ laser and a LED $(470 \mathrm{~nm})$ were used as the modulation and excitation sources, respectively.

\section{Results and discussion}

Room temperature PR and PL spectra for the $\mathrm{As}_{4}{ }^{-}$ -grown sample, L396, are shown in Fig. 1. The transition energies and broadening parameters were determined from fitting of the spectra to the first derivative of a Lorentzian-type function [4]. The PR spectrum typically shows four major sets of spectral features related to the various optical transitions in the QR structures investigated. The feature in the high-energy region at $1.67 \mathrm{eV}$ is attributed to a bandgap transition in the AlGaAs layer. Fitting of the spectrum yields a 20.5\% aluminium content, which is in good agreement with the $20 \%$ nominal value. For energies above the GaAs bandgap, an oscillating signal, resembling that of the Franz-Keldysh oscillations (FKO), appears. However, a plot of $4 / 3 \pi\left(E_{m}-E_{\mathrm{g}}\right)^{3 / 2}$ as a function of the extremum index number $m$ (not shown) does not give a straight line, as would be the case for the FKO effect. Therefore, we deduce that the PR oscillating signal is related to optical transitions between the above-barrier quasibound states localized in the GaAs layer of the QR structure. Indeed, the inclusion of the AlGaAs layer for better carrier confinement supports the formation of such quasibound above-barrier states. The energy interval below the bandgap of GaAs $(1.42 \mathrm{eV})$ corresponds to the optical transitions occurring in the InGaAs QW, surrounding the QRs. Finally, in the low-energy region of $1-1.2 \mathrm{eV}$, the PR spectrum exhibits broadened features related to the interband transitions in the InGaAs QRs. The later assignment is supported by PL data (Fig. 1), as well as by contactless electroreflectance (CER) spectroscopy [7].

Comparison of the PR and PL spectra related to the optical transitions occurring in the $\mathrm{QR}$ and $\mathrm{QW}$ regions for two similar $(N=20)$ structures - samples L396 and L402 - grown using $\mathrm{As}_{4}$ and $\mathrm{As}_{2}$ sources, respectively, is shown in Fig. 2. Energy values for interband optical transitions were deduced from fitting of the PR spectra to the first derivative of a Lorentzian-type function and are denoted by stars. The PR and PL features in the low energy region of 1-1.2 eV are red shifted by about $20 \mathrm{meV}$ for sample L396 compared to L402. The As 4 flux is thus accelerating Ga migration, leading to more indium-rich QRs, and giving lower energy emission [8]. This also explains the blue shift in Fig. 2 of about $10 \mathrm{meV}$ of QW transitions for sample L396 with respect to sample L402. In this case, use of $\mathrm{As}_{4}$ is increasing the indium composition contrast, $x_{\mathrm{QR}} / x_{\mathrm{QW}}$, leading to increased transition energy. It is also notable that the intensity of the PL signal is enhanced by a factor of two if an $\mathrm{As}_{4}$ source is used instead of $\mathrm{As}_{2}$. This enhancement is attributed to lower impurity incorporation into the epitaxial layer dur-

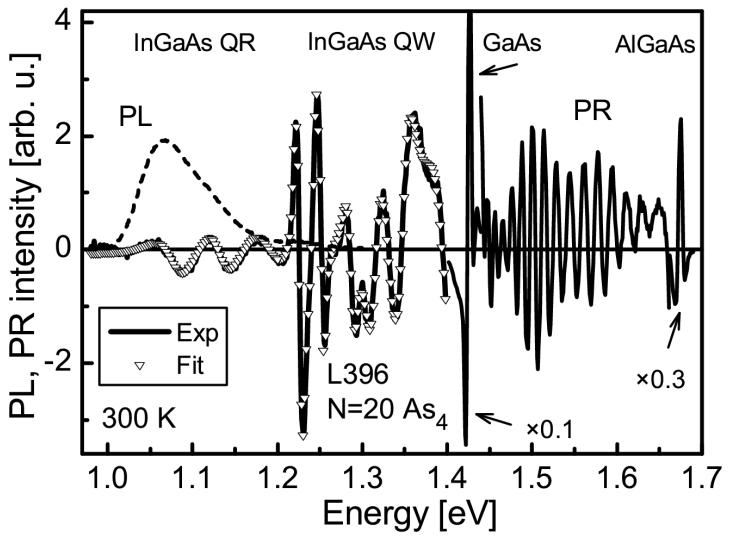

Fig. 1. Photoreflectance (PR) and photoluminescence (PL) spectra (solid and dashed curves, respectively) for sample L396 (As4 source) at room temperature. Open triangles represent fitting results using the first derivative of a Lorentzian-type function.

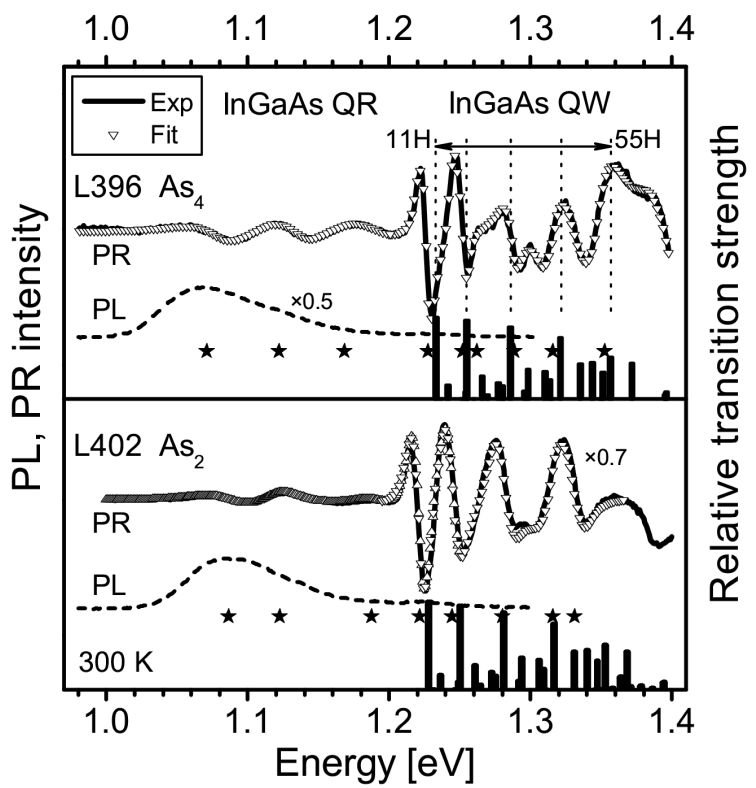

Fig. 2. Room temperature PR and PL spectra for the InGaAs QR structures with a 20-period SL (samples L396 and L402) grown using $\mathrm{As}_{4}$ (top) and $\mathrm{As}_{2}$ (bottom). Stars denote the interband transition energies determined from fitting of the PR spectra to the first derivative of a Lorentzian-type function (open triangles). Vertical bars indicate numerically evaluated [9] energies and relative strengths of the optical transitions in the InGaAs QW layer.

ing growth [8], owing to the cracker zone on the arsenic cell being cooler.

To obtain a deeper insight into the electronic structure of these intricate quantum systems, calculations were undertaken, based on an effective mass approximation, using the nextnano ${ }^{3}$ simulator [9], which has already successfully been applied for QD-superlattice structures 
$[5,10]$. It was assumed that the indium content $x_{\mathrm{QW}}$ follows the step-function at the material interfaces. The best qualitative agreement with experiment was then obtained if values of $x_{\mathrm{QW}}=0.175$ and $x_{\mathrm{QW}}=0.18$ were used for samples L396 and L402, respectively. The calculated optical transition energies and their relative strengths (overlap integrals) under zero electric field (flat bands) are indicated by vertical bars in Fig. 2. It was found that optical transitions between heavy-hole $(n)$ and electron bound states $(m)$ with the same quantum number $(n=m)$ dominate. However, the transitions with $n \neq m$ are also allowed due to the specific shape of the QW structures. Our calculations reveal that the blue shift of the QW-related optical spectrum for the $\mathrm{As}_{4}{ }^{-}$ grown sample with respect to the $\mathrm{As}_{2}$-grown sample can be attributed to variation in the indium content, $x_{\mathrm{QW}}$. As a consequence one can deduce that changes of the QR-related optical features in the PR and PL spectra (Fig. 2) for the two samples could also be associated with a variation of the indium content, $x_{\mathrm{QR}}$, depending on whether an $\mathrm{As}_{4}$ or $\mathrm{As}_{2}$ source is used.

To analyze the effect of indium content, $x_{\mathrm{QR}}$, on optical transitions originating from the QRs, we calculated the electronic states based on a model QR. Calculations were performed using a fully-3D approach taking into account strain and piezoelectric effects. The QR was assumed to have a cuboid shape with a height $h$ and a square base of length $d$. The key parameters were then varied as follows: $30 \leq h \leq 35 \mathrm{~nm}, 8 \leq d \leq 14 \mathrm{~nm}$, $0.45 \leq x_{\mathrm{QR}} \leq 0.6$ and $0.16 \leq x_{\mathrm{QW}} \leq 0.2$. From the simulations, the calculated energies were typically from 60 to $140 \mathrm{meV}$ above the measured energies for a given input set of parameters. The slight discrepancy between the calculated (not shown) and measured transition energies could be attributed to limitations of the effective mass approximation and excitonic effects. However, the behavior of calculated optical transitions agrees, in general, with the experimental observations well. In particular, it was found that the lowest-energy PR and PL features are due to transitions between heavy-hole and electron states, whilst higher-energy QR features involve light-hole related transitions. Finally, it is worth noting that the calculations predict the red shift of QR-related optical features owing to the formation of more indium-rich QR structure under an $\mathrm{As}_{4}$ flux.

\section{Conclusions}

In conclusion, PR and PL, together with numerical calculations based upon an effective mass approximation, were used to examine the optical properties and electronic structure of $\mathrm{QR}$ systems. It was found that the spectra of QR samples are very sensitive to the arsenic source used. A red shift of the QR- and a blue shift of the QW-related optical transitions, along with a significant PL signal intensity enhancement were obtained when an $\mathrm{As}_{4}$ was used instead of an $\mathrm{As}_{2}$ source.

\section{References}

[1] L.H. Li, G. Patriarche, M. Rossetti, A. Fiore, J. Appl. Phys. 102, 033502 (2007).

[2] J. Andrzejewski, G. Sek, E. O'Reilly, A. Fiore, J. Misiewicz, J. Appl. Phys. 107, 073509 (2010).

[3] P. Ridha, L.H. Li, M. Mexis, P.M. Smowton, J. Andrzejewski, G. Sek, J. Misiewicz, E.P. O'Reilly, G. Patriarche, A. Fiore, IEEE J. Quantum Electron. 46, 197 (2010).

[4] B. Čechavičius, J. Kavaliauskas, G. Krivaite, D. Seliuta, G. Valušis, M.P. Halsall, M.J. Steer, P. Harrison, J. Appl. Phys. 98, 023508 (2005).

[5] R. Nedzinskas, B. Čechavičius, J. Kavaliauskas, V. Karpus, D. Seliuta, V. Tamošiūnas, G. Valušis, G. Fasching, K. Unterrainer, G. Strasser, J. Appl. Phys. 106, 064308 (2009).

[6] L.H. Li, P. Ridha, G. Patriarche, N. Chauvin, A. Fiore, Appl. Phys. Lett. 92, 121102 (2008).

[7] M. Motyka, G. Sęk, K. Ryczko, J. Andrzejewski, J. Misiewicz, L.H. Li, A. Fiore, G. Patriarche, Appl. Phys. Lett. 90, 181933 (2007).

[8] L.H. Li, E.H. Linfield, S.P. Khanna, A.G. Davies, J. Appl. Phys. 108, 103522 (2010).

[9] nextnano ${ }^{3}$, next generation 3D nanodevice simulator, website: http://www.nextnano.de/nextnano3/.

[10] R. Nedzinskas, B. Čechavičius, J. Kavaliauskas, V. Karpus, G. Krivaitè, V. Tamošiūnas, G. Valušis, F. Schrey, K. Unterrainer, G. Strasser, Acta Phys. Pol. A 113, 975 (2008). 